\title{
Development of New Compact Water Treatment System for Flue-Gas Condenser for Households
}

\author{
Oskars SVEDOVS ${ }^{1 *}$, Mikelis DZIKEVICS ${ }^{2}$, Vladimirs KIRSANOVS ${ }^{3}$, Ivars VEIDENBERGS ${ }^{4}$ \\ ${ }^{1-4}$ Institute of Energy Systems and Environment, Riga Technical University, Azenes street 12/1, \\ Riga, LV-1048, Latvia
}

\begin{abstract}
This work is a continuation of the article "Methods for Determining the Performance and Efficiency Parameters of the Flue-gas Condenser Sedimentation Tank". During the experimental determination of Particulate Matter (PM) physical parameters, a described methodology was used. The results obtained affected the choice of water purification technology. Sedimentation technology was selected but provided that a filter element should be present in the installation. The article describes the types of industrial sedimentation tanks used more often. In condition that the system should be compact, while at the same time the purification efficiency rate should be high, the new suitable design of the water treatment system was developed.
\end{abstract}

Keywords - Fog Unit; particulate matter; sedimentation tank; water treatment

\begin{tabular}{|lll|}
\hline \multicolumn{2}{l}{ Nomenclature } & - \\
PM & Particulate Matter & - \\
BA & Bottom ash & - \\
FA & Fly ash & - \\
FU & Fog Unit - compact flue-gas treatment system & - \\
pH & Measure of acidity/basicity level & - \\
ST & Sedimentation tank & - \\
SS & Suspended solids/substances & pcs \\
$Q_{\text {PM }}$ & Quantity & - \\
CPMC & Compact particulate matter collector & \\
\hline
\end{tabular}

\section{INTRODUCTION}

The combustion of biomass results in energy, emissions, and ash. The ash is classified in two ways: bottom ash (BA) and fly ash (FA). The BA is the fraction formed as a result of the combustion of solid biomass fuels. The BA consists of the different salts, minerals, and also non-flammable or sand particles. The BA usually accumulates at the bottom of the combustion chamber. The FA are the particles that are finer than the BA and are therefore carried away with the flue-gases. FA consists mainly of Particulate Matter (PM) and heavy metals [1].

\footnotetext{
* Corresponding author.

E-mail address: oskars.svedovs@rtu.lv 
The presence of PM in the air adversely affects human health. Inhalation of air contaminated with PM primarily affects the human respiratory system. This may result in respiratory, cardiovascular diseases. Rare cases of reproductive and central nervous system dysfunction occur. Heavy metals, absorbing in the human body, cause poisoning or chronic intoxication. PM and heavy metals can also reach the human body from contaminated water. Living creatures or animals are also affected by PM heavy metals in the environment. Heavy metals can accumulate in several animal species. The long-term effects of toxic substances on soil or water can alter $\mathrm{pH}$ levels. As a result, environment may become unsuitable for the future functioning of the ecosystem [2].

The presence of PM in water can damage technical equipment, e.g., pumps. With time, the pump performance falls as it is designed for liquids. There are industrial water handling pumps with built-in shredders or particle collectors, but prolonged pollution impacts lead to clogging and critical impeller damage [3], [4].

PM causes damage to systems where a fluid is sprayed through a nozzle. A study by Azarov et al. [5] describes that there is a problem with the smallest nozzles. The smaller hole diameters of the modernized nozzles range from 5 to $10 \mu \mathrm{m}$. The $\mathrm{PM}_{2.5}$ passes through the nozzle, but larger particles can accumulate in the system. This leads to the clogging of the nozzle, leading to a deterioration in the technological process.

Riga Technical University's (RTU) Institute of Energy Systems and Environment in carrying out an experimental study of the wet flue-gas treatment system (scrubber) for households (Fog Unit (FU)) deals with water treatment problems. The water is circulating in the flue-gas treatment system as shown in Fig. 1. The water contaminated with PM flows out of the FU to a rectangular sedimentation tank (ST). It consists of three chambers: in the first chamber a primary sedimentation takes place, in the second chamber flows from heavier particles purified water, and in the third chamber a semi-purified water accumulates. Adding a ST in the system was a preventive measure to protect the pump and nozzle from damage. More detailed FU parameters are described in the previous article [6].

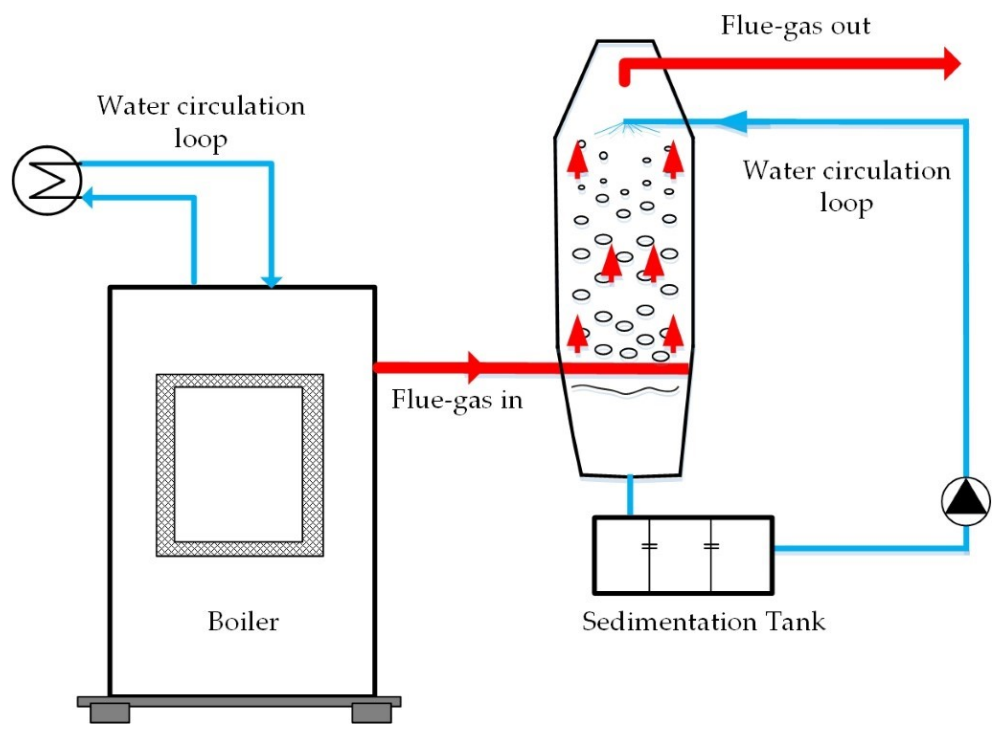

Fig. 1. Schematic of Fog Unit [6]. 
This system intended to be implemented in households; that is why a survey of respondents was carried out in the framework of the project. 157 respondents participated in the survey. Although mainly carried out concerning the operation and design of the FU, the survey results should be extended to the water purification aspect of the system. The results collected show that:

- $97.7 \%$ of respondents agree that the system must have simple maintenance;

- $95.3 \%$ of respondents think that electricity consumption should be as low as possible;

- $93.0 \%$ of respondents are concerned about the compact design of the system;

- $76.7 \%$ of respondents would like to collect the ash as easily as possible.

Among others, only $46.5 \%$ of respondents believe that the dry state of the ash collected is important [7].

Based on the results of the survey, the study aimed to improve the water treatment process in the FU system, to design and build an appropriate compact water treatment system. For this purpose, it was first necessary to investigate the physical parameters of PM, such as density, mass, and quantity distribution by size. This will mainly make it possible to understand which water purification technology is more suitable for the FU system.

\section{Determination of the Physical Parameters of the PM}

To determine the physical parameters of PM, a sample of the contaminated water was taken from the ST first chamber in FU system. Sampling was carried out following the steps described in ISO 56672. Suspended substances (SS) were obtained by drying a sample of water. An experimental study for this article is based on a methodology described by Svedovs et al. [6]. Photo and video materials were taken to determine the parameters using digital microscope equipment. The size and quantity of particles were determined by "ImageJ" software.

\subsection{Density Distribution by the PM Size}

The method of determining the density of the particles is based on Stokes Law [6]. PM density is possible to calculate by the equation:

$$
\rho_{p}=\frac{\mu \cdot 9 \cdot \omega}{g \cdot R^{2} \cdot 2}+\rho_{f},
$$

where

$\rho_{p} \quad$ Mass density of the $\mathrm{PM}, \mathrm{kg} / \mathrm{m}^{3}$;

$\rho_{f} \quad$ Mass density of the fluid, $\mathrm{kg} / \mathrm{m}^{3}$;

$\mu \quad$ Dynamic viscosity, $\mathrm{kg} /(\mathrm{m} \cdot \mathrm{s})$;

$\omega$ PM sedimentation velocity, $\mathrm{m} / \mathrm{s}$;

$R$ Radius of the PM, m;

$g$ Gravitational field strength, $\mathrm{m} / \mathrm{s}^{2}$.

Sedimentation velocity $\omega$ is calculated by the equation:

$$
\omega=\frac{s}{t}
$$

where $s$ is the distance travelled by the particle and $t$ is the travel time.

The radius of the $\mathrm{PM}$ is calculated by the equation: 


$$
R=\sqrt{\frac{S_{p}}{\pi}},
$$

where $S_{p}$ is the particle area and $\pi$ is the constant ratio of a circle's circumference to its diameter.

The density was determined for each particle as shown in Eq. (1). Because of the different size and chemical composition, each particle has a bulk density rather than an absolute density.

The resulting experimental data were collected and processed. As can be seen in Fig. 2, most particles have a density that is less than water density $\left(997 \mathrm{~kg} / \mathrm{m}^{3}\right)$. The results can be intercepted in a way that doesn't sink such particles into the water because they have the negative sedimentation time. That means they aspire to the surface of the water. Fig. 2 shows that there are PM with a radius of $10 \mu \mathrm{m}$ and larger. The filter element should be incorporated into the ST [6]. It will be possible to hold particles in the system. On the other hand, particles with the radius $10 \mu \mathrm{m}$ and less have the positive sedimentation time.

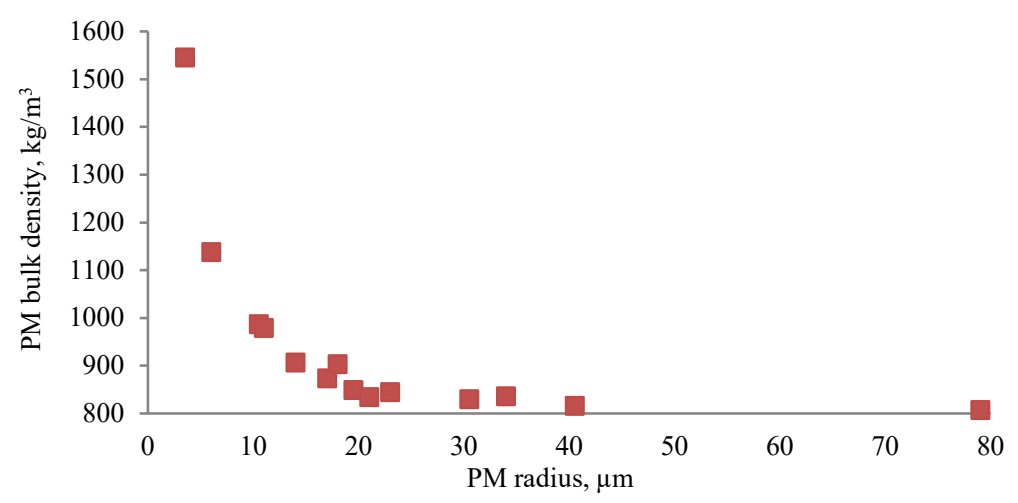

Fig. 2. Determined PM bulk density dependency on PM radius.

\subsection{Quantity Distribution by the PM Size}

The quantity distribution by the size typically reflects the relative content of particles of different sizes in soil. In water chemistry, it may be used to assess the distribution of particles in SS by size.

The microscope's digital equipment was used to get several pictures of PM. The 'ImageJ' software was used to analyze 10 randomly selected pictures. Each picture showing a real $1 \mathrm{~mm}^{2}$ area. For quicker analyses, particles were divided by radius ranges. The PM that was taken into account were particles with a radius of $0.5 \mu \mathrm{m}$ and larger.

As can be seen in Fig. 3, the most subtle PM with a radius range of $0.5-1 \mu \mathrm{m}$ are found. On average, there are $312 \mathrm{pcs} / \mathrm{mm}^{2}$. The average quantity of PM in the radius range $1-10 \mu \mathrm{m}$ is $177 \mathrm{pcs} / \mathrm{mm}^{2}$. There are $68 \mathrm{pcs} / \mathrm{mm}^{2}$ in the radius range $10-25 \mu \mathrm{m}$. There are very few PM with a radius of $25 \mu \mathrm{m}$ or more.

Since the study did not include all particles, the standard deviation is calculated for the selection. Consequently, certain limits of credibility are established within each range of particle radius. Standard deviation in all subsequent schedules is indicated by black error lines. 


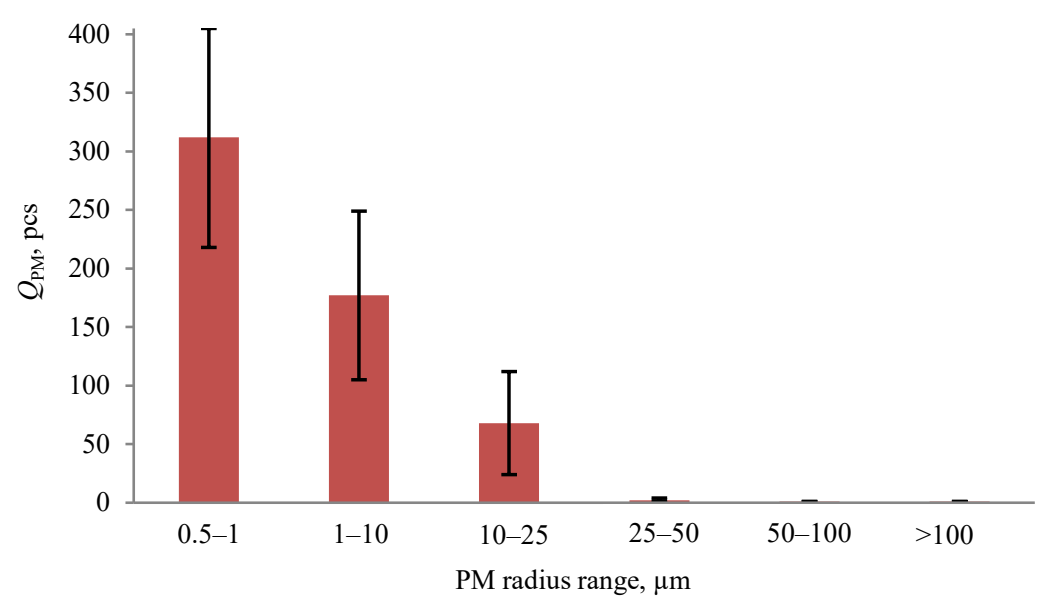

Fig. 3. Counted PM quantity dependency on PM radius ranges.

\subsection{Mass Distribution by the PM Size}

It is possible to use the determined PM density $\rho_{p}$ for the calculation of particle mass. Assuming the particle has a sphere shape, the mass can be calculated by the equation:

$$
m=\left(\frac{4}{3} \cdot \pi \cdot R^{3}\right) \cdot \rho_{p},
$$

where

$m$ Mass of the PM, kg;

$R \quad$ Radius of the PM, m;

$\rho_{p} \quad$ Mass density of the PM, $\mathrm{kg} / \mathrm{m}^{3}$;

$\pi \quad$ Ratio of a circle's circumference to its diameter, constant.

The average mass of one particle over each radius range was calculated as shown in Eq. (5). The total PM mass $m_{t}$ was calculated by the equation:

$$
m_{t}=m \cdot Q_{\mathrm{PM}},
$$

where $m$ is the mass of the PM and $Q_{\mathrm{PM}}$ is the quantity of PM.

As can be seen in Fig. 4, a large quantity of PM (312 pcs) with a radius in the range from $0.5-1 \mu \mathrm{m}$ have an average weight of $0.0007 \mu \mathrm{g}$. Each PM with a radius ranges of $50-100 \mu \mathrm{m}$ and $>100 \mu \mathrm{m}$ weights on average $2.21 \mu \mathrm{g}$ and $3.93 \mu \mathrm{g}$ respectively. It is a relatively high mass compared to the particles with a smaller radius ranges included in the study, but the amount of these particles is well below. In eight cases out of ten, no particles with a radius ranges of $50-100 \mu \mathrm{m}$ and $>100 \mu \mathrm{m}$ were found. In such a case, it may be considered that the largest mass of the total distribution is composed of PM with a radius range of $10-25 \mu \mathrm{m}$. The average total mass of this PM is $2.04 \mu \mathrm{g}$. 


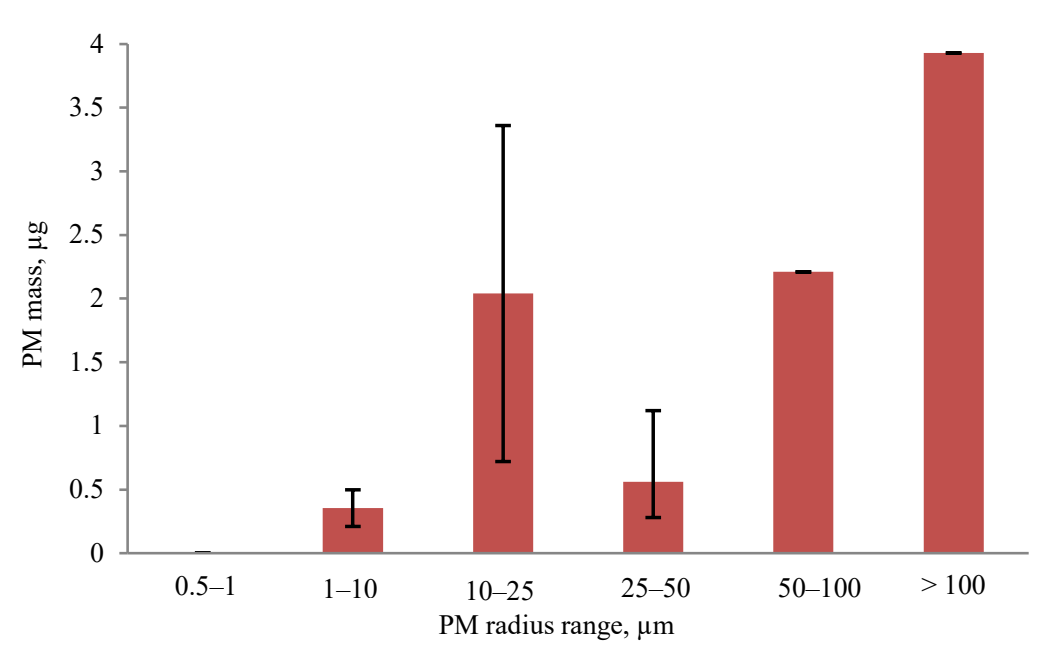

Fig. 4. Determined PM mass dependency on PM radius ranges.

\subsection{Conclusions from the Experimental Part}

The results of the measurements are used to choose the water purification technology. PM with a radius of less than $10 \mu \mathrm{m}$ is identified as having a positive sedimentation time in the water and PM with the radius of more than $10 \mu \mathrm{m}$ having negative sedimentation time in the water. Moreover, the number of PM with the radius of less than $10 \mu \mathrm{m}$ is higher. To sink into the water, such PM will precipitate independently.

PM with the radius of more than $10 \mu \mathrm{m}$ has a greater total mass, but they cannot precipitate independently in water. The sedimentation process may take place if additional forces are applied. If such PM are given an acceleration, they will be able to reach a larger path (directed downwards) due to inertia during the sedimentation process.

\section{Development Process of the Compact Particle Matter COLLECTOR}

\subsection{Overview of Water Purification Technologies}

Water chemistry offers a variety of purification technologies with a wide range of applications. For example, multi-cartridge, cartridge and hydrocyclone type filters, water purification systems with granulated active coal, etc. The most common method of treatment of industrial wastewater is sedimentation. The process is mostly cheap, with low operating and maintenance costs, because sedimentation is provided by physical forces (gravity and hydromechanical forces), but therefore the process is slow [8], [9].

One of the most popular types of ST is a continuous settler. It can be rectangular (the simplest design) or cylindrical. The sedimentation process starts in the first chamber in which most of the SS precipitate. After a time, the water is released into the next chamber in which the process is repeated [10]. As has already been mentioned in the article, this kind of ST is currently in use in the FU system as shown in Fig. 1. The purified water is reused in the fluegas treatment system. As a result of the repeated use of water, such a solution is not appropriate in the short term. 
The cylindrical continuous settler works on the same principle, but it differs constructively. It is usually a closed system with a cover to prevent contamination from external sources. water flow is directed in a radial direction. Each of the three cylindrical cameras has the prescribed dimensions. The first camera is the smallest. It mainly serves as an inlet tube that helps to convert turbulent water flow into laminar. The sedimentation process begins in the second chamber. The SS precipitate at the bottom of the second chamber and water flows into the next chamber in a radial direction. In the last chamber, the sedimentation process recurs, the water flows into the output channel and is removed from the system. The cylindrical continuous settler structure provides for faster and higher-quality water treatment [10].

Another type of cylindrical ST that many companies offer on the market operates on the principle of water vortex (hydrocyclone). It is widely used in the urban sewer system to collect larger solid objects, sludge, and SS such as sand pulp, from industrial wastewater or waters that come into the sewer with rain or melting snow [8].

Constructively, the hydrocyclone or swirl separator is made cylindrical with a conical basis for removal of SS. A study by Ochowiak et al. [8] describes that the tank's height and inlet tube position have less impact on the separation performance. The greatest importance is the geometry of inlet and outlet pipes and the diameter of the tank. The authors point out that the removal of SS is due to gravity rather than centrifugal force. Hydrocyclone can also function effectively at compact sizes. The design ensures effective removal of SS at high hydraulic loads.

The structure of the radial flow circular ST is similar to that of the hydrocyclone, but the water flow hydraulics are different. It is widely applied in cases where municipal wastewater contains a large amount of sludge. In Fig. 5, structural differences between hydrocyclone (a) and radial flow circular ST (b) can be seen: both designs differ mainly with the inlet tube placement (1), while the water outlet tube to the drumfilter (2), the purified water outlet tube (3) and the suspended discharge tube (4) remain in the same places. In hydrocyclone, the water flow depends on the centrifugal force tank inside. Therefore, the water inlets tangentially. In the radial flow circular ST water enters the central inner chamber to ensure the sedimentation of the SS and the flow of water in a radial direction. The central chamber is needed to convert the water flow from the turbulent into the laminar. The radial flow circular ST has a lower hydraulic load [11].

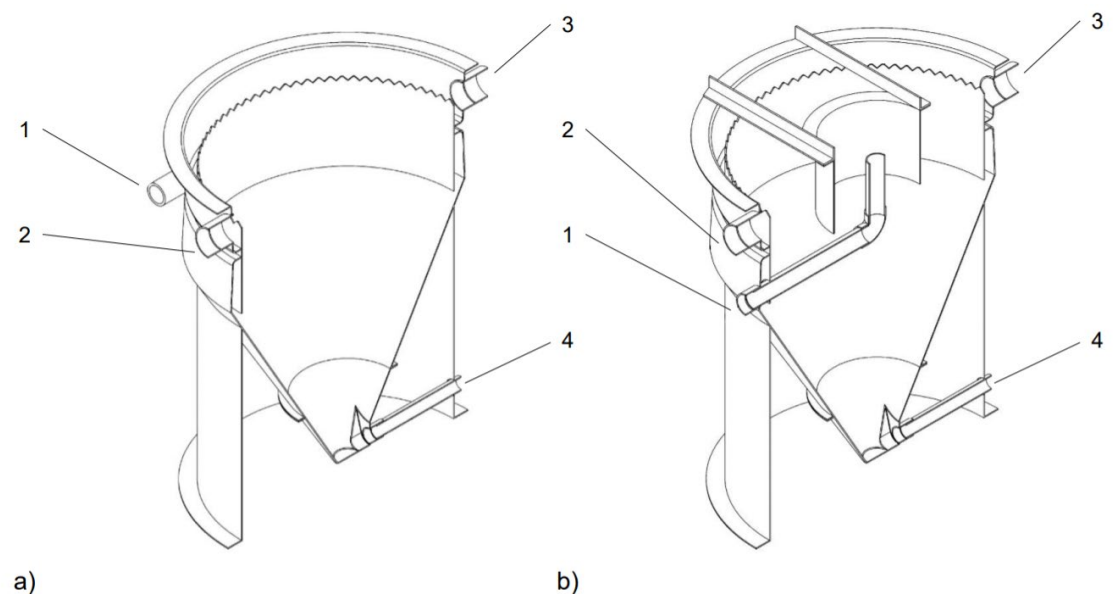

Fig. 5. Line drawings of the hydrocyclone (a) and radial flow sedimentation tank (b) [11]. 
Ochowiak et al. [8] mentions that choosing the right type of ST will affect efficiency gains. In the case of water contaminated only with SS (in our case - with PM), the use of a radial flow circular ST is more appropriate in terms of treatment efficiency.

In an overview of the literature on this subject concluded that the method of settling the SS from the liquid is used more often.

\subsection{System Design and Its Explanation}

The compact particulate matter collector (CPMC) system is intended for the treatment of ash slurries (suspension) - for the removal of the solid fraction (ash dust) from the liquid fraction (water) used in the flue-gas treatment process. It was therefore decided to base the principle of the operation of the CPMC on the principle of the functioning of the ST.

A radial sedimentation tank was selected as the basis for a prototype of a new water treatment system. The choice was made in such a way that, firstly, the water purified by the radial sedimentation tank flows in a radial direction and, secondly, there is an overflow problem in the one-way continuous settler.

The methodology for creating a design of the CPMC is based on experimental analysis of PM physical parameters. The CPMC must be equipped with a filtering element because most of the PM in the study have a lower density than water, and therefore their sedimentation time in the water is negative (not downwards, but upward). As a result, it is necessary to stop the flow of PM in water. The following applies to PM with a radius value of $10 \mu \mathrm{m}$ or more. For finer PM, the density value is greater than water density, the sinking exists, respectively. Therefore, the filter element mesh should be as small as possible.

If the CPMC system is fitted with a pump, the Stokes Law will not work because of additional force (Stokes Law works on natural settlement due to gravity). In this case, it is important to know the mass flow vector to predict PM behaviour into CPMC. It was concluded that most of the total mass is composed of PM larger than $10 \mu \mathrm{m}$. Most of the total mass flow in the inlet will be downward due to inertia. The CPMC must be equipped with a storage chamber from which it will be possible to remove the collected PM.

CPMC (Fig. 6) consists of suspension inlet tube (1), purified water outlet channel (2) and outlet tube (3), particle storage chamber (4), filter element mesh (5) and the stored particle drainage tube (6).

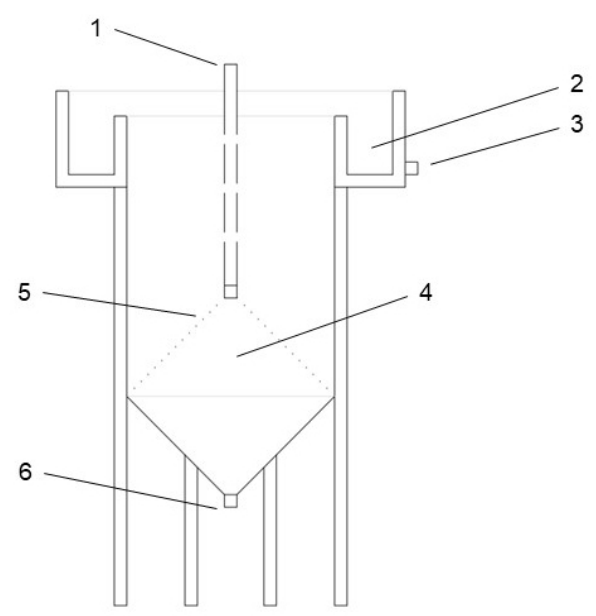

Fig. 6. The main CPMC components providing the water purification process. 


\subsection{Final Design Development Process}

A 3D printer approach was used for the prototyping of the CPMC. The 3D model was developed with 'Autodesk Inventor Professional 2020'. The final model was created using a 3D printer. Acrylonitrile butadiene styrene (ABS plastic) was used as a material. 3D printer nozzle size was $2 \mathrm{~mm}$. The total model volume is approximately 49 litres. The limitations of the printer required splitting the prototype into units of $300 \mathrm{~mm} \times 300 \mathrm{~mm}$.

Six separate components of the final model were developed (Fig. 7): cover part (1), purified water outlet channel (2), suspension inlet tube (3), settling chamber (4), filter element mesh (5) and particle storage chamber (6).

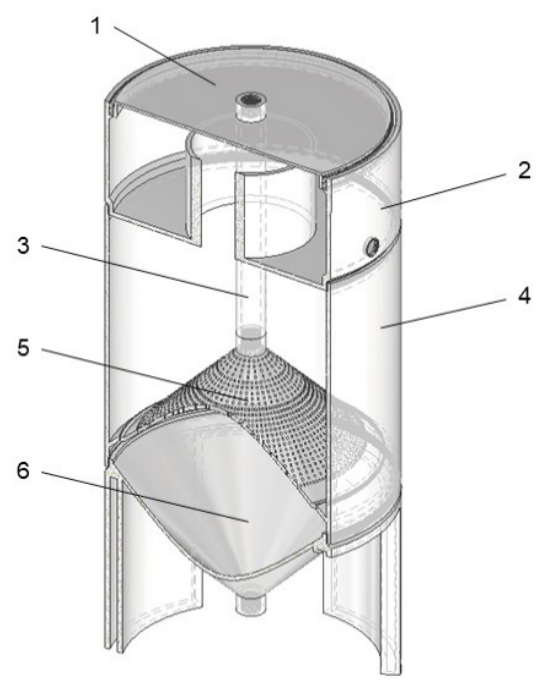

Fig. 7. Components of the CPMC prototype 3D model.

The settling chamber is the basic part of the CPMC in which the settling process takes place. Threads were created to connect parts to the settling chamber providing option of separation and modularity. The volume of the settling chamber is approximately 21 litres.

The particle storage chamber has upper and lower parts. The upper part serves as a filter element with mesh. Threads were created for attaching related parts to the filter element. The limitations of 3D printing allowed the minimum hole size for the filter element of $2 \mathrm{~mm}$. The lower part of the particle storage chamber consists of support legs and a drainage hole. It will be accompanied by a valve and drainage tube. The settling process in the direction of the drainage hole is provided by an angle of $45^{\circ}$. The volume of the particle storage chamber is approximately 10 litres.

The water separated from the PM flows from the settling chamber into the purified water outlet channel, which is connected to the settling chamber. It will be accompanied by the valve and water outlet tube. The pump may be inserted at this stage of the system. The volume of the purified water outlet channel is approximately 18 litres.

The suspension inlet tube is inserted into the central hole of the cover part and screwed to the filter element. When contaminated water flows through this tube, the SS will partially precipitate immediately into the particle storage chamber lower part. The water flow is directed upward through a filter element mesh that holds PM in the chamber. 


\subsection{Theoretical Calculation of PM Retention Time}

For rectangular ST with the horizontal flow, performance is characterized by multiplying the sedimentation area with the known SS sedimentation time. Accordingly, the sedimentation area can be calculated to achieve the required performance. For other types of ST, more complex calculations performed.

It is possible to compare different types of ST by SS retention time parameter. In general, retention time $T$ is calculated by the equation:

$$
T=\frac{V_{s}}{\dot{Q}_{w}},
$$

where $V_{s}$ is the volume of settling zone and $Q_{w}$ is volumetric water flow rate.

The theoretical retention time for the CPMC is calculated for multiple flow rates (Fig. 8). The volume of the settling zone for CPMC is 10 litres equal to the volume of the particle storage chamber. Water flow values are taken from FU testing. Fig. 8 shows that at 50 1/hour the PM retention time is $720 \mathrm{~s}$. As the flow increases to $2501 /$ hour, the PM retention time decreases to $72 \mathrm{~s}$.

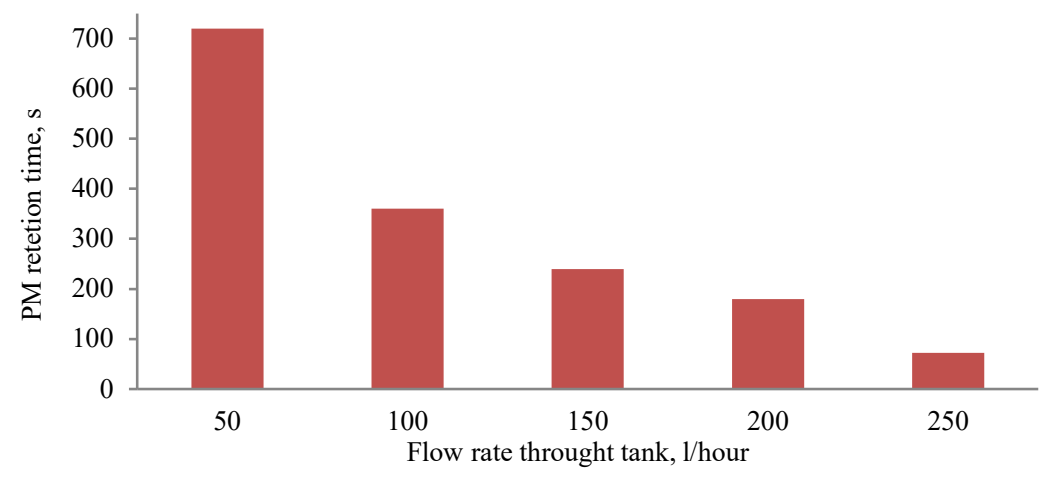

Fig. 8. Theoretical PM retention time in the CPMC depending on the flow rate.

\section{Conclusion}

The design of the CPMC includes the functionality of the ST and filter. It is necessary to capture PM in the water treatment system. The results of the survey carried out in the context of the Fog Unit project considered in the design of the CPMC, providing convenient assembly and disassembly in household conditions. Therefore, it will be easy to maintain the CPMC.

Difficulties were encountered in the process of creating a prototype. The $3 \mathrm{D}$ printing method used in this study proved to be unsatisfactory regarding the threads that could be created lacking in precision and durability. The threaded option was selected to make it possible to disassemble the unit therefore would be crucial to make it work in the final product. Either a more precise printer of latch system should be considered in future iterations to provide leak-proof connection and ease of maintenance.

Due to the specificity of the 3D printing and the physical properties of the ASB plastic, the prototype had poor durability. The suspension inlet tube, particle storage chamber outlet tube and legs were damaged during the building of an experimental stand requiring to be fixed with epoxy 
resin. This means that the technology chosen (3D printing) to build a prototype is inappropriate. It would be more appropriate to make a prototype of metal.

An experimental study of the CPMC prototype is planned. The main aim is to determine the water purification efficiency of CPMC. In addition, it is planned to clarify the effects of the PM concentration, turbidity, $\mathrm{pH}$ level and water conductivity on the functioning of the installation.

\section{ACKNOWLEDGEMENT}

The research is funded by the Ministry of Economics of the Republic of Latvia, project "Assessment of Latvia's renewable energy supply-demand economic potential and policy recommendations", Project No. VPP-EM-2018/AER-10001. The result was achieved in cooperation with the RTU Design Factory.

\section{REFERENCES}

[1] Agrela F., et al. Biomass fly ash and biomass bottom ash. New Trends in Eco-efficient and Recycled Concrete. Woodhead Publishing Series in Civil and Structural Engineering 2019:23-58. https://doi.org/10.1016/B978-0-08-102480-5.00002-6

[2] Manisalidis I., et al. Environmental and Health Impacts of Air Pollution: A Review. Frontiers in Public Health 2020:8:14. https://doi.org/10.3389/fpubh.2020.00014

[3] Wilson K. C., et al. Effect of Solids on Pump Performance BT - Slurry Transport Using Centrifugal Pumps. Boston: Springer US 2006:227-248.

[4] Merkle T. Introduction. Damages on Pumps and Systems:The Handbook for the Operation of Centrifugal Pumps. Amsterdam: Elsevier, 2014.

[5] Azarov A. V., Zhukova N. S., Antonov F. G. Water-spray systems reducing negative effects of finedispersion dust at operator's workplaces of machine-building industries. Procedia Engineering 2017:206:1407-1414. https://doi.org/10.1016/j.proeng.2017.10.653

[6] Švedovs O., Dzikēvičs M., Kirsanovs V. Methods for Determining the Performance and Efficiency Parameters of the Flue-gas Condenser Sedimentation Tank. Environmental and Climate Technologies 2020:24(2):337-347. https://doi.org/10.2478/rtuect-2020-0077

[7] Priedniece V., et al. Treatment of Particulate Matter Pollution: People's Attitude and Readiness to Act. Environmental and Climate Technologies 2020:24(2):231-246. https://doi.org/10.2478/rtuect-2020-0069

[8] Ochowiak M., et al. The modified swirl sedimentation tanks for water purification. Journal of Environmental Management 2017:189:22-28. https://doi.org/10.1016/j.jenvman.2016.12.023

[9] Khorrami Z., Banihashemi M. A. Numerical simulation of sedimentation process in reservoirs and development of a non-coupled algorithm to improve long-term modeling. International Journal of Sediment Research 2019:34(3):279-294. https://doi.org/10.1016/j.ijsrc.2018.10.003

[10] Luna F. T. D. et al. Fluid dynamics in continuous settler. Chemical Engineering Journal 2018:362:712720. https://doi.org/10.1016/j.cej.2019.01.088

[11] Davidson J., Summerfelt S. T. Solids removal from a coldwater recirculating system - Comparison of a swirl separator and a radial-flow settler. Aquacultural Engineering 2005:33(1):47-61. https://doi.org/10.1016/j.aquaeng.2004.11.002 